\title{
Rehabilitationssport - Aktivierung ist nicht gleich Therapie
}

Nach § 26 SGB IX hat ein Arzt die Möglichkeit, eine Verordnung für Rehabilitationssport und Funktionstraining auszustellen (SGB 2004). Ziele dieser Leistungen zur medizinischen Rehabilitation Behinderter und von Behinderung bedrohter Menschen sind einerseits, Behinderungen einschließlich chronischer Krankheiten abzuwenden, zu beseitigen, zu mindern, auszugleichen oder eine Verschlimmerung $\mathrm{zu}$ verhüten. Andererseits sollten Einschränkungen der Erwerbsfähigkeit und Pflegebedürftigkeit abgewendet, überwunden oder vermindert, sowie eine Verschlimmerung verhütet werden, damit der vorzeitige Bezug von laufenden Sozialleistungen vermieden oder laufende Sozialleistungen vermindert wird. Ursprünglich waren diese Maßnahmen zur Aktivierung behinderter Menschen mit den vorher erwähnten Zielen gedacht, werden aber heute auch bei Menschen mit chronischen Muskelskelettbeschwerden angewendet. Das gezielte und gut lokalisierte Training, abgestimmt auf den einzelnen Patienten, geht hierbei verloren, weil aus wirtschaftlichen Gründen in großen Gruppen mit Teilnehmern, denen unterschiedlichste Diagnosen gestellt wurden, gearbeitet werden muss. Der therapeutische Wert reduziert sich dann lediglich auf den Aspekt der Aktivierung. 